\title{
The Pohozaev-type inequalities and their applications for a kind of elliptic equation (system)
}

\author{
Bingyu Kou ${ }^{1 *}$ (D), Tianqing $\mathrm{An}^{2}$ and Zeyan Wang ${ }^{1}$
}

"Correspondence: koubei@163.com 'Department of Basic Courses, The Army Engineering University of PLA, Nanjing, 211101, P.R. China Full list of author information is available at the end of the article

\begin{abstract}
In this paper, we first derive a new kind of Pohozaev-type inequalities for p-Laplacian equations in a more general class of non-star-shaped domains, and then we take two examples and their graphs to explain the shape of the new kind of the non-star-shaped domain. At last, we extend the results of Pohozaev-type inequalities to elliptic systems, which are used to derive the nonexistence of positive solutions of this type of systems in the non-star-shaped domains. On this basis, we also discuss the existence of positive solutions of a kind of elliptic systems with double critical growth.

MSC: 35J50; 35J92

Keywords: Pohozaev-type inequality; p-Laplacian; Non-star-shaped domain; Variational methods; Nonexistence and existence; Double critical exponent; Ekeland variational principle
\end{abstract}

\section{Introduction}

In this paper, we first consider the following elliptic equation:

$$
\begin{cases}-\Delta_{p} u=f(u), & \text { in } \Omega, \\ u=0, & \text { on } \partial \Omega\end{cases}
$$

where $\Delta_{p}=\operatorname{div}\left(|\nabla u|^{p-2} \nabla u\right), f \in C\left(R^{1}, R^{1}\right)$, and $\Omega \subset \mathbb{R}^{n}(n \geq 3)$ is a domain with smooth boundary. We establish the Pohozaev-type inequalities not Pohozaev identities for the solutions of (1.1), and by which we derive the nonexistence of positive solutions for the problem in non-star-shaped domains. Subsequently, we take two examples and their graphs to explain the shape of the new kind of the non-star-shaped domain. Then, we also consider a similar problem for the following elliptic system:

$$
\begin{cases}-\operatorname{div}\left(|\nabla u|^{p-2} \nabla u\right)=f_{u}(u, v) & x \in \Omega, \\ -\operatorname{div}\left(|\nabla v|^{p-2} \nabla v\right)=f_{v}(u, v) & x \in \Omega, \\ u=v=0 & x \in \partial \Omega,\end{cases}
$$

(c) The Author(s) 2020. This article is licensed under a Creative Commons Attribution 4.0 International License, which permits use, sharing, adaptation, distribution and reproduction in any medium or format, as long as you give appropriate credit to the original author(s) and the source, provide a link to the Creative Commons licence, and indicate if changes were made. The images or other third party material in this article are included in the article's Creative Commons licence, unless indicated otherwise in a credit line to the material. If material is not included in the article's Creative Commons licence and your intended use is not permitted by statutory regulation or exceeds the permitted use, you will need to obtain permission directly from the copyright holder. To view a copy of this licence, visit http://creativecommons.org/licenses/by/4.0/. 
where $\Delta_{p}=\operatorname{div}\left(|\nabla u|^{p-2} \nabla u\right), f_{u}(u, v)=\frac{\partial F(u, v)}{\partial u}, f_{v}(u, v)=\frac{\partial F(u, v)}{\partial v}, f_{u}(u, v), f_{v}(u, v): \mathbb{R} \times \mathbb{R} \rightarrow \mathbb{R}$, and they are continuous. $\Omega \subset \mathbb{R}^{N}(N \geq 3)$ is also a domain with smooth boundary.

When $p=2$ in (1.1), Pohozaev proved the following well-known Pohozaev identity in the famous paper [1]:

$$
(2-n) \int_{\Omega} u f(u) d x+2 n \int_{\Omega} F(u) d x=\int_{\partial \Omega}\langle x, v(x)\rangle\left|\frac{\partial u}{\partial v}\right|^{2} d s
$$

Based on the identity, Pohozaev, Brezis, and Nirenberg considered the following problem in $[1,2]$ :

$$
\begin{cases}-\Delta u=|u|^{p-1} u+\lambda u & x \in \Omega, \\ u>0 & x \in \Omega, \\ u=0 & x \in \partial \Omega\end{cases}
$$

where $\lambda \in \mathbb{R}, \Omega \subset \mathbb{R}^{N}(N \geq 3)$ is a bounded domain with $C^{1}$ boundary. They proved that if $\Omega \subset \mathbb{R}^{N}(N \geq 3)$ is a star-shaped bounded domain, problem (1.4) has no solution when both $p \geq \frac{n+2}{n-2}, \lambda \leq 0$ and $p>\frac{n+2}{n-2}, \lambda \leq \delta(\Omega, p)$.

Subsequently, Kawano and $\mathrm{Ni}$ discussed the general semi-linear elliptic equation when $p=2$ in [3]:

$$
\begin{cases}-\Delta_{p} u=f(x, u) & x \in \Omega, \\ u>0 & x \in \Omega, \\ u=0 & x \in \partial \Omega .\end{cases}
$$

By the classic Pohozaev proof method, the authors derived the following identity:

$$
\begin{aligned}
(2 & -n) \int_{\Omega} u f(x, u) d x+2 n \int_{\Omega} F(x, u) d x+2 \int_{\Omega}\left(x, F_{x}(x, u)\right) d x \\
& =\int_{\partial \Omega}\langle x, v(x)\rangle\left|\frac{\partial u}{\partial v}\right|^{2} d s .
\end{aligned}
$$

In 1989, Guedda and Veron considered (1.1) in [4] and got the following identity:

$$
(p-n) \int_{\Omega} u f(u) d x+p n \int_{\Omega} F(u) d x=(p-1) \int_{\partial \Omega}\langle x, v(x)\rangle\left|\frac{\partial u}{\partial v}\right|^{p} d s,
$$

where $F(u)=\int_{0}^{u} f(t) d t, f(u)$, is continuous. And they got the nonexistence results of nontrivial solutions in the case of supercritical growth and critical growth when $1<p<N$ and $\Omega \subset \mathbb{R}^{N}$ is a star-shaped domain about origin. See [5-11] and the references therein. In [12], we established more general Pohozaev-type inequalities of (1.5) and got the nonexistence of positive solutions when $\Omega \subset \mathbb{R}^{N}$ is a non-star-shaped domain.

In this paper, we discuss more general Pohozaev-type inequalities and the nonexistence of positive solutions of (1.1). At the same, we take two examples and their graphs to explain the shape of a new kind of the non-star-shaped domain.

The existence or nonexistence of nontrivial positive solutions of elliptic system (1.2) have been extensively studied in recent years, we refer to [13-16] and the references 
therein. Researchers usually convert the proof of the existence of nonlinear elliptic problems into finding the existence of the critical point of the variational functional corresponding to the equation, in which, one of the key issues is to prove that the (PS) sequence has a strongly convergent sub-sequence. Therefore, the first step is to prove the boundedness of the (PS) sequence. Scholars have put forward many conditions such as the famous A-R condition. However, the A- $R$ condition cannot be satisfied in many cases. In recent years, it was shown that combining the Pohozaev identity and the Nehari manifold to create a new manifold, which is called Pohozaev-Nehari manifold, was very effective when treating nonlinearity which does not satisfy the A-R condition (see [17-19]).

In [17], Li and Ye considered the following Kirchhoff equation:

$$
\begin{cases}-\left(a+b \int_{\mathbb{R}^{3}}|\nabla u|^{2} d x\right) \Delta u+u=|u|^{p-1} u & x \in \mathbb{R}^{3}, \\ u \in H^{1}\left(\mathbb{R}^{3}\right), \quad u>0 & x \in \mathbb{R}^{3} .\end{cases}
$$

They established the Pohozaev identity

$$
P(u):=\frac{a}{2} \int_{\mathbb{R}^{3}}|\nabla u|^{2} d x+\frac{3}{2} \int_{\mathbb{R}^{3}}|u|^{2} d x+\frac{b}{2}\left(\int_{\mathbb{R}^{3}}|\nabla u|^{2} d x\right)^{2}-\frac{3}{p+1} \int_{\mathbb{R}^{3}}|u|^{p+1} d x
$$

and the new Pohozaev-Nehari manifold

$$
\mathcal{M}=\left\{u \in H^{1}\left(\mathbb{R}^{3}\right) \backslash 0 \mid G(u)=0\right\},
$$

where $G(u)=\left\langle I^{\prime}(u), u\right\rangle+P(u)$. The authors proved that the new manifold (1.8) was $C^{1}$ and the critical point of the limit equation on the new manifold was the critical point in the entire space. Subsequently, the solution of the equation was obtained by finding the least energy solution of the limit equation of the corresponding equation in the new manifold.

In [19], the authors studied the existence of a bound state solution for a class of quasilinear Schrödinger equations whose nonlinear term is asymptotically linear in $\mathbb{R}^{N}$. The equation is as follows:

$$
-\triangle u+V(x) u-\triangle\left(u^{2}\right) u=g(u), \quad x \in \mathbb{R}^{N}, N \geq 3,
$$

where $V(x)=W(x)-E$ is the new potential and $g(u)=l\left(u^{2}\right) u$ is the nonlinear term. The authors defined the following Pohozaev manifold:

$$
\mathcal{P}=\left\{v \in H^{1}\left(\mathbb{R}^{N}\right) \backslash 0 \mid \gamma(v)=0\right\}
$$

and

$$
\mathcal{P}_{\infty}=\left\{v \in H^{1}\left(\mathbb{R}^{N}\right) \backslash 0 \mid v \text { satisfies Pohozaev identity }\right\},
$$

where

$$
\begin{aligned}
\gamma(v)= & \frac{N-2}{2} \int_{\mathbb{R}^{N}}|\nabla v|^{2} d x+\frac{N}{2} \int_{\mathbb{R}^{N}} V(x) f^{2}(v) d x+\frac{1}{2} \int_{\mathbb{R}^{N}}(\nabla V(x), x) f^{2}(v) d x \\
& -N \int_{\mathbb{R}^{N}} G(f(v)) d x,
\end{aligned}
$$


and the corresponding Pohozaev identity of the limiting problem is as follows:

$$
\frac{N-2}{2} \int_{\mathbb{R}^{N}}|\nabla v|^{2} d x+\frac{N}{2} \int_{\mathbb{R}^{N}} V_{\infty} f^{2}(v) d x=N \int_{\mathbb{R}^{N}} G(f(v)) d x .
$$

Employing the minimization methods restricted to the above Pohozaev manifold, the authors obtained the existence of positive solutions for the asymptotically linear case.

In [16], Bozhkov and Mitidieri studied the existence and nonexistence of the following (p,q)-Laplacian system:

$$
\begin{cases}-\Delta_{p} u=\lambda a(x)|u|^{p-2} u+(\alpha+1) c(x)|u|^{\alpha-1} u|v|^{\beta+1} & x \in \Omega, \\ -\Delta_{q} v=\mu b(x)|v|^{q-2} v+(\beta+1) c(x)|u|^{\alpha+1}|v|^{\beta-1} v & x \in \Omega, \\ u=v=0 & x \in \partial \Omega .\end{cases}
$$

The authors also generalized the Pohozaev identity for the following system which is based on reference [20]:

$$
\begin{cases}-\operatorname{div}\left(|\nabla u|^{p-2} \nabla u\right)=F_{u}(x, u, v) & x \in \Omega, \\ -\operatorname{div}\left(|\nabla v|^{q-2} \nabla v\right)=F_{v}(x, u, v) & x \in \Omega, \\ u=v=0 & x \in \partial \Omega .\end{cases}
$$

And the Pohozaev identity is as follows:

$$
\begin{aligned}
& \frac{N-p}{p} \int_{\Omega}|\nabla u|^{p} d x+\frac{N-q}{q} \int_{\Omega}|\nabla v|^{q} d x-N \int_{\Omega} F(x, u, v) d x-\int_{\Omega} D_{x} F(x, u, v) d x \\
& =-\left(1-\frac{1}{p}\right) \int_{\partial \Omega}|\nabla u|^{p}(x, v) d s-\left(1-\frac{1}{q}\right) \int_{\partial \Omega}|\nabla v|^{q}(x, v) d s .
\end{aligned}
$$

By this kind of Pohozaev identity, the authors derived the nonexistence of the positive solutions on the star-shaped domain. However, on the basis of preliminary work of Pohozaev inequalities for $\mathrm{p}$-Laplacian equation, we can generalize the kind of identity to the kind of Pohozaev inequalities for the p-Laplace system and (p,q)-Laplacian systems, and naturally get the nonexistence of these systems on the non-star-shaped domain, which is more general than the domain in [16]. In addition, we consider the following elliptic system with double critical growth:

$$
\begin{cases}-\Delta_{p} u=|u|^{p^{*}-2} u+\frac{\alpha}{p^{*}}|u|^{\alpha-2}|v|^{\beta} u, & x \in \Omega, \\ -\Delta_{p} v=|v|^{p^{*}-2} v+\frac{\beta}{p^{*}}|u|^{\alpha}|v|^{\beta-2} v, & x \in \Omega, \\ u=v=0, & x \in \partial \Omega, \\ u>0, \quad v>0 & x \in \Omega,\end{cases}
$$

where $\alpha, \beta>1, \alpha+\beta=p^{*}=\frac{N p}{N-p}$. Using the Pohozaev inequalities, we can prove that system (1.11) has no positive solution when $\Omega$ is a positive domain. It is worth to mention that the more general Pohozaev to the general elliptic equations in a non-star-shaped bounded domain can help us to obtain the necessary conditions of the existence of solutions for some special equations. See [21-27]. In [27], Peng, Peng, and Wang discussed in detail 
the existence and the form of positive solution, the uniqueness of the minimal energy solution of system (1.11) when $p=2$. Based on their global compactness result and the main method in [21], we consider the existence of positive solution of system (1.11) for general $p$.

\section{The results and proofs}

We need the following lemma, which is similar but also has some differences with our manuscript [12].

Lemma 2.1 Suppose $V(x)=\left(V_{1}(x), \ldots, V_{n}(x)\right)$, which is a $C^{1}$ vector field on $\mathbb{R}^{n}$ and $u \in$ $W_{0}^{1, p}(\Omega) \cap C^{1}(\bar{\Omega})$ is a solution of $(1.1)$. Then

$$
\int_{\Omega} u \operatorname{div}\left(|\nabla u|^{p-2} V(x)\right) d x=-\int_{\Omega}|\nabla u|^{p-2}\langle V(x), \nabla u\rangle d x
$$

and

$$
\int_{\Omega} F(u) \operatorname{div} V(x) d x=-\int_{\Omega} f(u)\langle V(x), \nabla u\rangle d x .
$$

Based on the above lemma, we can have a Pohozaev-type inequality for solutions of equation (1.1).

Theorem 2.2 (Pohozaev-type inequality) Suppose that $V(x)$ is a linear vector field on $\mathbb{R}^{n}$ with the form

$$
V(x)=\left(\begin{array}{ccc}
a_{11} & \cdots & a_{1 n} \\
\vdots & \vdots & \vdots \\
a_{n 1} & \cdots & a_{n n}
\end{array}\right) x
$$

and $V(x)$ satisfies $\operatorname{div} V(x)=n$ and $\langle V(x), x\rangle>0$ for $\forall x \in \mathbb{R}^{n} \backslash\{0\}$. If $u \in W_{0}^{1, p}(\Omega) \cap C^{1}(\bar{\Omega})$ is a solution of (1.1), then

$$
\begin{gathered}
(p \mu-n) \int_{\Omega} u f(u) d x+p n \int_{\Omega} F(u) d x \\
\geq(p-1) \int_{\partial \Omega}\langle V(x), v(x)\rangle\left|\frac{\partial u}{\partial v}\right|^{p} d s
\end{gathered}
$$

where $\mu=\sup _{|x| \neq 0} \frac{\langle V(x), x\rangle}{|x|^{2}}$.

Proof From $\mu=\sup _{|x| \neq 0} \frac{\langle V(x), x\rangle}{|x|^{2}}$, we easily get

$$
0<\langle V(x), x\rangle<\mu|x|^{2}, \quad \forall x \in \mathbb{R}^{n} \backslash\{0\} .
$$

Multiply the equation $-\Delta_{p} u=f(u)$ by $\langle V(x), \nabla u\rangle$ and then integrate in $\Omega$. By Lemma 2.1 and divergence theorem, the right-hand side is

$$
-\int_{\Omega} f(u)\langle V(x), \nabla u\rangle d x=-\int_{\Omega} \sum_{i=1}^{n} f(u) V_{i}(x) \frac{\partial u}{\partial x_{i}}=\int_{\Omega} F(u) \operatorname{div} V(x) d x,
$$


and then the left-hand side is

$$
\begin{aligned}
& \int_{\Omega} \operatorname{div}\left(|\nabla u|^{p-2} \nabla u\right)\langle V(x), \nabla u\rangle d x \\
& \quad=\sum_{j=1}^{n} \int_{\Omega} \frac{\partial}{\partial x_{j}}\left(|\nabla u|^{p-2} \frac{\partial u}{\partial x_{j}}\right)\left(\sum_{i=1}^{n} V_{i}(x) \frac{\partial u}{\partial x_{i}}\right) d x \\
& =\sum_{j=1}^{n} \int_{\Omega} \frac{\partial}{\partial x_{j}}\left(|\nabla u|^{p-2} \frac{\partial u}{\partial x_{j}} \sum_{i=1}^{n} V_{i}(x) \frac{\partial u}{\partial x_{i}}\right)-|\nabla u|^{p-2} \frac{\partial u}{\partial x_{j}} \frac{\partial}{\partial x_{j}}\left(\sum_{i=1}^{n} V_{i}(x) \frac{\partial u}{\partial x_{i}}\right) d x .
\end{aligned}
$$

We also have

$$
\begin{aligned}
& \int_{\Omega} \operatorname{div}\left(|\nabla u|^{p-2} \nabla u\right)\langle V(x), \nabla u\rangle d x \\
& =\int_{\Omega} \operatorname{div}\left(|\nabla u|^{p} V(x)\right) d x \\
& -\sum_{j=1}^{n} \int_{\Omega}|\nabla u|^{p-2} \frac{\partial u}{\partial x_{j}}\left(\sum_{i=1}^{n} a_{i j} \frac{\partial u}{\partial x_{i}}+\sum_{i=1}^{n} V_{i}(x) \frac{\partial^{2} u}{\partial x_{i} \partial x_{j}}\right) \\
& =\int_{\partial \Omega}\left\langle V(x),\left.v(x)|| \nabla u\right|^{p} d s\right. \\
& -\sum_{j=1}^{n} \int_{\Omega}|\nabla u|^{p-2} \frac{\partial u}{\partial x_{j}}\left(\sum_{i=1}^{n} a_{i j} \frac{\partial u}{\partial x_{i}}+\sum_{i=1}^{n} V_{i}(x) \frac{\partial^{2} u}{\partial x_{i} \partial x_{j}}\right) \\
& =\int_{\partial \Omega}\left\langle V(x),\left.v(x)|| \nabla u\right|^{p} d s+\int_{\Omega} u \operatorname{div}\left(|\nabla u|^{p-2} V(\nabla u)\right)\right. \\
& -\sum_{j=1}^{n} \int_{\Omega}|\nabla u|^{p-2} \frac{\partial u}{\partial x_{j}}\left(\sum_{i=1}^{n} V_{i} \frac{\partial^{2} u}{\partial x_{i} \partial x_{j}} \frac{\partial u}{\partial x_{j}}\right) \\
& =\int_{\partial \Omega}\left\langle V(x),\left.v(x)|| \nabla u\right|^{p} d s-\int_{\Omega}|\nabla u|^{p-2}\langle V(\nabla u), \nabla u\rangle d x\right. \\
& -\frac{1}{2} \sum_{i=1}^{n} \int_{\Omega}|\nabla u|^{p-2} V_{i}(x) \frac{\partial}{\partial x_{i}}\left(|\nabla u|^{2}\right) d x \\
& =\int_{\partial \Omega}\left\langle V(x),\left.v(x)|| \nabla u\right|^{p} d s-\int_{\Omega}|\nabla u|^{p-2}\langle V(\nabla u), \nabla u\rangle d x\right. \\
& -\frac{1}{p} \sum_{i=1}^{n} \int_{\Omega} V_{i}(x) \frac{\partial}{\partial x_{i}}\left(|\nabla u|^{p}\right) d x \\
& =\int_{\partial \Omega}\left\langle V(x),\left.v(x)|| \nabla u\right|^{p} d s-\int_{\Omega}|\nabla u|^{p-2}\langle V(\nabla u), \nabla u\rangle d x\right. \\
& -\frac{1}{p} \int_{\partial \Omega}\left\langle V(x),\left.v(x)|| \nabla u\right|^{p} d s+\frac{1}{p} \sum_{i=1}^{n} a_{i i} \int_{\Omega}|\nabla u|^{p} d x .\right.
\end{aligned}
$$

Comparing the left- and right-hand sides, we know the following identity:

$$
\left(1-\frac{1}{p}\right) \int_{\partial \Omega}|\nabla u|^{p}\langle V(x), v(x)| d s
$$




$$
\begin{aligned}
& =-\frac{1}{p} \sum_{i=1}^{n} a_{i i} \int_{\Omega}|\nabla u|^{p} d x+\int_{\Omega}|\nabla u|^{p-2}\langle V(\nabla u), \nabla u\rangle d x+\int_{\Omega} F \operatorname{div} V(x) d x \\
& =-\frac{1}{p} \sum_{i=1}^{n} a_{i i} \int_{\Omega} u f(u) d x+\int_{\Omega}|\nabla u|^{p-2}\langle V(\nabla u), \nabla u\rangle d x+\sum_{i=1}^{n} a_{i i} \int_{\Omega} F(u) d x .
\end{aligned}
$$

By the fact $0<\langle V(x), x\rangle \leq \mu|x|^{2}$, we get the following result:

$$
0<\langle V(\nabla u), \nabla u\rangle \leq \mu|\nabla u|^{2} .
$$

Thus,

$$
\int_{\Omega}|\nabla u|^{p-2}\left\langle V(\nabla u),\left.\nabla u\left|d x \leq \mu \int_{\Omega}\right| \nabla u\right|^{p} d x=\mu \int_{\Omega} u f(u) d x\right.
$$

because $\operatorname{div} V(x)=\sum_{i=1}^{n} a_{i i}=n$, we obtain the following inequality:

$$
\begin{gathered}
(p \mu-n) \int_{\Omega} u f(u) d x+p n \int_{\Omega} F(u) d x \\
\geq(p-1) \int_{\partial \Omega}\langle V(x), v(x)\rangle\left|\frac{\partial u}{\partial v}\right|^{p} d s .
\end{gathered}
$$

The proof is complete.

By the above Pohozaev-type inequality, we can immediately get the nonexistence result of the following problem:

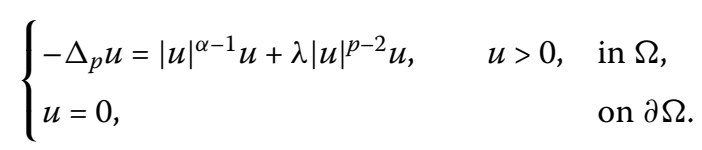

Theorem 2.3 Assume that the vector field $V(x)$ of Theorem 2.2 is transverse to $\partial \Omega$. Then (2.2) has no positive solution if

$$
\alpha \geq \frac{n p-n+p \mu}{n-p \mu} \text { and } \lambda \leq 0 .
$$

Proof With the similar method in the proof of Theorem 2.2, we also first consider that (1.1) has a positive solution $u$. Then inequality (2.1) holds,

$$
\begin{aligned}
& (p \mu-n) \int_{\Omega}\left(u^{\alpha+1}+\lambda u^{p}\right) d x+p n \int_{\Omega}\left(\frac{1}{\alpha+1} u^{\alpha+1}+\frac{\lambda}{p} u^{p}\right) d x \\
& \geq(p-1) \int_{\partial \Omega}\langle V(x), v(x)\rangle\left|\frac{\partial u}{\partial v}\right|^{p} d s .
\end{aligned}
$$

That is,

$$
\begin{aligned}
& \left(p \mu-n+\frac{p n}{\alpha+1}\right) \int_{\Omega} u^{\alpha+1} d x+p \mu \lambda \int_{\Omega} u^{p} d x \\
& \geq \int_{\partial \Omega}\langle V(x), v(x)\rangle\left|\frac{\partial u}{\partial v}\right|^{p} d s .
\end{aligned}
$$


Since $\alpha \geq \frac{n p-n+p \mu}{n-p \mu}$, we have $p \mu-n+\frac{p n}{\alpha+1} \leq 0$. Thus,

$$
p \mu \lambda \int_{\Omega} u^{p} d x \geq \int_{\partial \Omega}\langle V(x), v(x)\rangle\left|\frac{\partial u}{\partial v}\right|^{p} d s .
$$

If $\lambda<0,(2.3)$ contradicts the fact $\langle V(x), v(x)\rangle>0$.

If $\lambda=0,(2.3)$ implies that $\frac{\partial u}{\partial v}=0$. By divergence theorem,

$$
\begin{aligned}
\int_{\Omega} \Delta_{p} u d x & =\int_{\Omega} \operatorname{div}\left(|\nabla u|^{p-2} \nabla u\right) d x \\
& =\int_{\partial \Omega}\left\langle|\nabla u|^{p-2} \nabla u, v\right\rangle d s=\int_{\partial \Omega}|\nabla u|^{p-2} \frac{\partial u}{\partial v} d s=0 .
\end{aligned}
$$

On the other hand, the equation in (2.2) implies

$$
\int_{\Omega} \Delta_{p} u d x=-\int_{\Omega} u|u|^{\alpha-1} d x<0,
$$

which is a contraction. Then, we complete the proof.

By the way, we consider a special case of $p=2$ which was discussed in [28]. Take $p=2$ in Theorem 2.2 and Theorem 2.3, we have the following conclusions.

Corollary 2.4 (Pohozaev-type inequality for $p=2$ ) Suppose that $V(x)$ is a linear vector field on $\mathbb{R}^{n}$ satisfying $\operatorname{div} V(x)=n$ and $\langle V(x), x\rangle>0$ for $\forall x \in \mathbb{R}^{n} \backslash\{0\}$. If $u \in W_{0}^{1, p}(\Omega) \cap C^{1}(\bar{\Omega})$ is a solution of (1.1) when $p=2$, then

$$
\begin{gathered}
(2 \mu-n) \int_{\Omega} u f(u) d x+2 n \int_{\Omega} F(u) d x \\
\geq \int_{\partial \Omega}\langle V(x), v(x)\rangle\left|\frac{\partial u}{\partial v}\right|^{2} d s
\end{gathered}
$$

where $\mu=\sup _{|x| \neq 0} \frac{\langle V(x), x\rangle}{|x|^{2}}$.

Corollary 2.5 Suppose that the vector field $V(x)$ of Corollary 2.4 is transverse to $\partial \Omega$. Then (1.4) has no positive solution if

$$
\alpha \geq \frac{n+2 \mu}{n-2 \mu} \text { and } \quad \lambda \leq 0 .
$$

\section{Examples}

In this section, we construct two examples of domains in $\mathbb{R}^{3}$ which satisfy the conditions of our theorems but not non-star-shaped. The first example has been discussed in [28], the second one is new. 


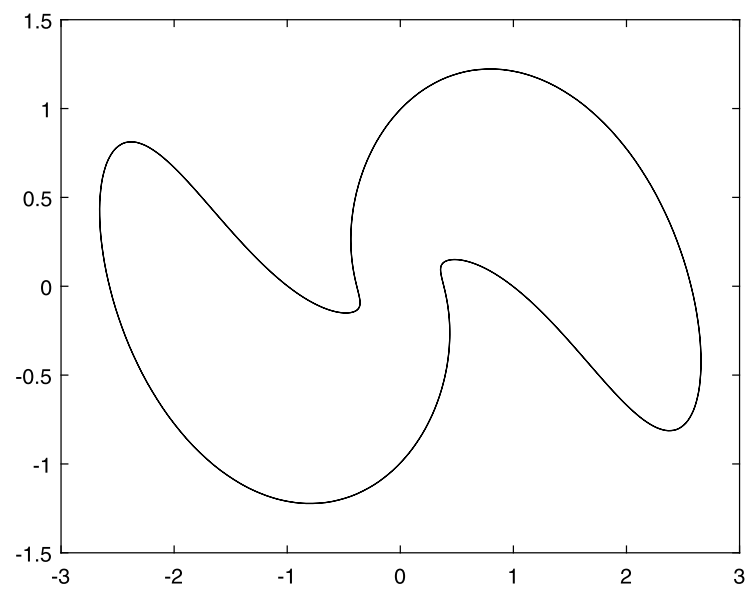

Figure 1 The graph of $\Gamma_{1}$

Example 1 (see [28]) Let $\Omega_{1} \subset \mathbb{R}^{3}$ be a bounded domain surrounded by the following closed surface $\Sigma_{1}$ :

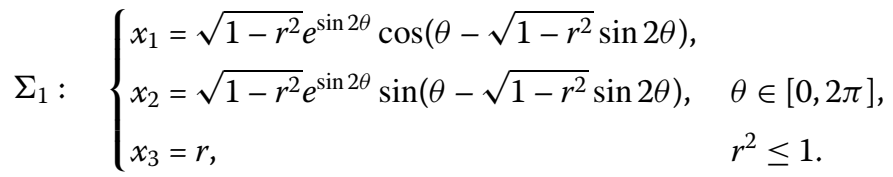

In [28], the second author of the paper has proved that, for any given point $P \in \mathbb{R}^{3}$, the closed surface $\Sigma_{1}$ is not star-shaped with respect to $P$. Thus, $\Omega_{1}$ is not star-shaped. Subsequently, it is easy to verify that $\Omega_{1}$ satisfies the conditions of Theorem 2.2.

Next, we will give the specific graphic of the planar closed curve $\Gamma_{1}$ in [28], which is generated by the intersection of $\Sigma_{1}$ and $\Pi$, where $\Pi=\left\{x=\left(x_{1}, x_{2}, 0\right) \mid x_{1} \in \mathbb{R}^{1}, x_{2} \in \mathbb{R}^{1}\right\}$. Figure 1 is the graph of $\Gamma_{1}$.

Example 2 Let $\Omega_{2} \subset \mathbb{R}^{3}$ be a bounded domain surrounded by the following closed surface $\Sigma_{2}$ :

$$
\Sigma_{2}: \begin{cases}x_{1}=\sqrt{1-r^{2}} e^{\cos 2 \theta} \cos \left(\theta+\frac{1}{2} \sqrt{1-r^{2}} \sin 4 \theta\right), & \\ x_{2}=\sqrt{1-r^{2}} e^{\cos 2 \theta} \sin \left(\theta+\frac{1}{2} \sqrt{1-r^{2}} \sin 4 \theta\right), & \theta \in[0,2 \pi], \\ x_{3}=r, & r^{2} \leq 1 .\end{cases}
$$

Define a linear vector field $V$ on $\mathbb{R}^{3}$ by

$$
V(x)=\left(\begin{array}{ccc}
\frac{3}{7} & 0 & 0 \\
0 & \frac{15}{7} & 0 \\
0 & 0 & \frac{3}{7}
\end{array}\right) x
$$

It is easy to see that $V$ is positive and $\sum_{i=1}^{3} a_{i i}=3$. By a direct computation, we can show that $V(x)$ is transverse to $\Sigma_{2}$. Therefore, $\Omega_{2}$ satisfies the conditions of Theorem 2.2. 


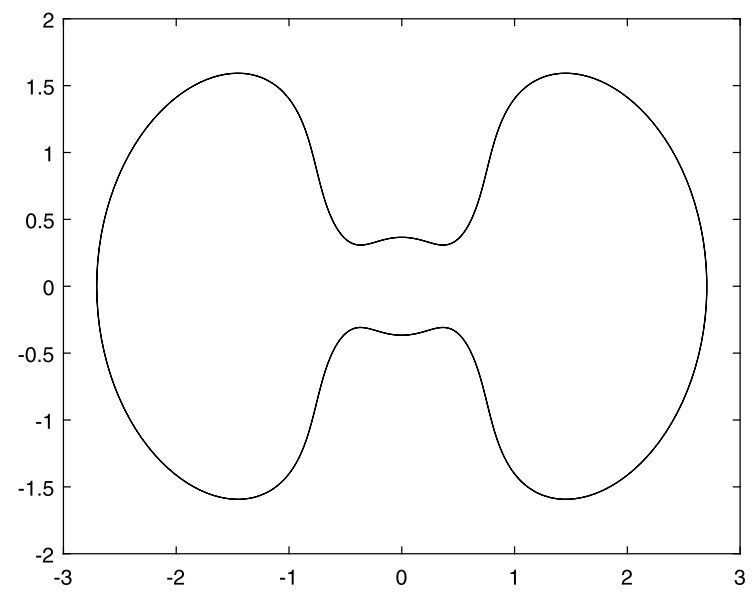

Figure 2 The graph of $\Gamma_{2}$

Let $\Gamma_{2}$ be the curve given by the intersection of plane $\Pi=\left\{x=\left(x_{1}, x_{2}, 0\right) \mid x_{1} \in \mathbb{R}^{1}, x_{2} \in\right.$ $\left.\mathbb{R}^{1}\right\}$ and $\Sigma_{2}$ :

$$
\Gamma_{2}:\left\{\begin{array}{l}
x_{1}=e^{\cos 2 \theta} \cos \left(\theta+\frac{1}{2} \sin 4 \theta\right), \\
x_{2}=e^{\cos 2 \theta} \sin \left(\theta+\frac{1}{2} \sin 4 \theta\right), \quad \theta \in[0,2 \pi] \\
x_{3}=0 .
\end{array}\right.
$$

The graph of $\Gamma_{2}$ is presented in Fig. 2.

By the elementary method, we can show that $\Gamma_{2}$ is not star-shaped in the plane. Hence, $\Omega_{2}$ is not star-shaped.

\section{The Pohozaev inequalities and the nonexistence of positive solutions for p-Laplace elliptic systems}

In this section, we generalize the results of Pohozaev inequalities of Theorem 2.2 to system (1.2) to derive the nonexistence of positive solutions for the system with double critical exponent. Firstly, define the following Sobolev space:

$$
\widetilde{W}_{\Omega}=W_{0}^{1, p}(\Omega) \times W_{0}^{1, p}(\Omega)
$$

with the norm

$$
\|(u, v)\|=\left(\int_{\Omega}|\nabla u|^{p}+|\nabla v|^{p}\right)^{\frac{1}{p}}
$$

Applying Theorem 2.2 to system (1.2), we get the following theorem. 
Theorem 4.1 (Pohozaev inequalities) Assume that $V(x)$ is a linear vector field on $\mathbb{R}^{N}$ of the form

$$
V(x)=\left(\begin{array}{ccc}
a_{11} & \cdots & a_{1 n} \\
\vdots & \vdots & \vdots \\
a_{n 1} & \cdots & a_{n n}
\end{array}\right) x
$$

for $\forall x \in \mathbb{R}^{N} \backslash\{0\}, V(x)$ satisfies $\operatorname{div} V(x)=N$ and $\langle V(x), x\rangle>0$. If $(u, v) \in \widetilde{W}(\Omega) \cap C^{1}(\bar{\Omega})$ is a solution of (1.2), then

$$
\begin{gathered}
(p \mu-N) \int_{\Omega}(u, v) \nabla F(u, v) d x+p N \int_{\Omega} F(u, v) d x \\
\geq(p-1) \int_{\partial \Omega}\langle V(x), v(x)\rangle\left(\left|\frac{\partial u}{\partial v}\right|^{p}+\left|\frac{\partial v}{\partial v}\right|^{p}\right) d s,
\end{gathered}
$$

where $\mu=\sup _{|x| \neq 0} \frac{\langle V(x), x\rangle}{|x|^{2}}$.

Proof Applying Theorem 2.2 to the first equation of system (1.2), we have

$$
\begin{gathered}
(p \mu-N) \int_{\Omega} u f_{u}(u, v) d x+p N \int_{\Omega} F_{u}(u, v) d x \\
\geq(p-1) \int_{\partial \Omega}\langle V(x), v(x)\rangle\left|\frac{\partial u}{\partial v}\right|^{p} d s .
\end{gathered}
$$

Similarly, applying Theorem 2.2 to the second equation of system (1.2), we have

$$
\begin{gathered}
(p \mu-N) \int_{\Omega} v f_{v}(u, v) d x+p N \int_{\Omega} F_{v}(u, v) d x \\
\geq(p-1) \int_{\partial \Omega}\langle V(x), v(x)\rangle\left|\frac{\partial v}{\partial v}\right|^{p} d s .
\end{gathered}
$$

Adding (4.2) and (4.3), we obtain the desired result.

Similarly, applying Theorem 2.2 and Theorem 2.2 in [12] to two equations in the system of (1.9), we can generalize the Pohozaev identity (1.10) of (p,q)-Laplace system in [16] to Pohozaev inequalities.

Theorem 4.2 (Pohozaev inequalities) Suppose that $V(x)$ is a linear vector field on $\mathbb{R}^{N}$, and let

$$
V(x)=\left(\begin{array}{ccc}
a_{11} & \cdots & a_{1 n} \\
\vdots & \vdots & \vdots \\
a_{n 1} & \cdots & a_{n n}
\end{array}\right) x .
$$


$\forall x \in \mathbb{R}^{N} \backslash\{0\}, V(x)$ satisfies $\operatorname{div} V(x)=N$ and $\langle V(x), x\rangle>0$. If $(u, v) \in \widetilde{W}(\Omega) \cap C^{1}(\bar{\Omega})$ is a solution of (1.9), then

$$
\begin{aligned}
& \frac{(p \mu-N)}{p} \int_{\Omega}(u, v) \nabla F(x, u, v) d x+\frac{(q \mu-N)}{q} \int_{\Omega}(u, v) \nabla F(x, u, v) d x \\
& \quad+N \int_{\Omega} F(x, u, v) d x+\int_{\Omega} F_{1}(x, u, v) d x \\
& \geq\left(1-\frac{1}{p}\right) \int_{\partial \Omega}\langle V(x), v(x)\rangle\left|\frac{\partial u}{\partial v}\right|^{p} d s+\left(1-\frac{1}{q}\right) \int_{\partial \Omega}\langle V(x), v(x)\rangle\left|\frac{\partial v}{\partial v}\right|^{q} d s,
\end{aligned}
$$

where $\mu=\sup _{|x| \neq 0} \frac{\langle V(x), x\rangle}{|x|^{2}}$.

Proof Based on Theorem 2.2 and [12], and the equations of system (1.9), we have

$$
\begin{aligned}
& (p \mu-N) \int_{\Omega} u F_{u}(x, u, v) d x+p N \int_{\Omega} F_{u}^{1}(x, u, v) d x+p \int_{\Omega} F_{1, u}(x, u, v) d x \\
& \geq(p-1) \int_{\partial \Omega}\langle V(x), v(x)\rangle\left|\frac{\partial u}{\partial v}\right|^{p} d s
\end{aligned}
$$

and

$$
\begin{aligned}
& (q \mu-N) \int_{\Omega} v F_{v}(x, u, v) d x+q N \int_{\Omega} F_{v}^{1}(x, u, v) d x+q \int_{\Omega} F_{1, v}(x, u, v) d x \\
& \geq(q-1) \int_{\partial \Omega}\langle V(x), v(x)\rangle\left|\frac{\partial v}{\partial v}\right|^{q} d s,
\end{aligned}
$$

where $\frac{\partial F_{u}^{1}(x, u, v)}{\partial u}=f_{u}(x, u, v)$, and $\frac{\partial F_{v}^{1}(x, u, v)}{\partial u}=f_{v}(x, u, v)$. Adding (4.5) and (4.6), we complete the proof.

Remark 1 The Pohozaev inequalities in Theorem 4.2 can also be written in the following forms:

$$
\begin{aligned}
& \frac{(p \mu-N)}{p} \int_{\Omega}|\nabla u|^{p} d x+\frac{(q \mu-N)}{q} \int_{\Omega}|\nabla v|^{q} d x+N \int_{\Omega} F(x, u, v) d x+\int_{\Omega} F_{1}(x, u, v) d x \\
& \geq\left(1-\frac{1}{p}\right) \int_{\partial \Omega}\left\langle V(x),\left.v(x)|| \nabla u\right|^{p} d s+\left(1-\frac{1}{q}\right) \int_{\partial \Omega}\left\langle V(x),\left.v(x)|| \nabla v\right|^{q} d s .\right.\right.
\end{aligned}
$$

Now, we discuss the nonexistence of positive solutions for system (1.11).

Theorem 4.3 Suppose that the vector field $V(x)$ of Theorem 4.1 is transverse to $\partial \Omega$. Then (1.11) has no positive solution.

Proof Let $F(u, v)=\frac{1}{p^{*}} \int_{\Omega}\left(|u|^{p^{*}}+|v|^{p^{*}}+|u|^{\alpha}|v|^{\beta}\right) d x$, by Theorem 4.3, the right-hand side of the Pohozaev inequalities can be written as follows:

$$
\left(1-\frac{1}{p}\right) \int_{\partial \Omega}\langle V(x), v(x)\rangle\left(\left|\frac{\partial u}{\partial v}\right|^{p}+\left|\frac{\partial v}{\partial v}\right|^{p}\right) d s \geq 0
$$


the left-hand side is

$$
\begin{aligned}
& =\left(\mu-\frac{N}{p}\right) \int_{\Omega}\left(|\nabla u|^{p}+|\nabla v|^{p}\right) d x+\frac{N}{p^{*}} \int_{\Omega}\left(|u|^{p^{*}}+|v|^{p^{*}}+|u|^{\alpha}|v|^{\beta}\right) d x \\
& \leq\left(\mu-\frac{N}{p}\right) \int_{\Omega}\left(|\nabla u|^{p}+|\nabla v|^{p}\right) d x \\
& <0 .
\end{aligned}
$$

This is a contradiction, so (1.11) has no positive solution.

\section{The existence of positive solutions for a kind of p-Laplace elliptic system}

This section studies the existence of positive solutions of (1.11).

Consider the functional $J: \widetilde{W}_{\Omega} \rightarrow \mathbb{R},(u, v) \in \widetilde{W}_{\Omega}$,

$$
J(u, v)=\frac{1}{p} \int_{\Omega}\left(|\nabla u|^{p}+|\nabla v|^{p} d x\right)-\frac{1}{p^{*}} \int_{\Omega}\left(\left(u^{+}\right)^{p^{*}}+\left(v^{+}\right)^{p^{*}}+\left(u^{+}\right)^{\alpha}\left(v^{+}\right)^{\beta}\right) d x,
$$

and $(u, v) \in \widetilde{W}_{\mathbb{R}^{N}}$,

$$
J^{\infty}(u, v)=\frac{1}{p} \int_{\mathbb{R}^{N}}\left(|\nabla u|^{p}+|\nabla v|^{p} d x\right)-\frac{1}{p^{*}} \int_{\mathbb{R}^{N}}\left(\left(u^{+}\right)^{p^{*}}+\left(v^{+}\right)^{p^{*}}+\left(u^{+}\right)^{\alpha}\left(v^{+}\right)^{\beta}\right) d x .
$$

By Theorem 1.7 in [27] and Lemma 3.4.5 in [29], we have the global compactness results as follows.

Lemma 5.1 (Global compactness result, see [27]) Let $\left(u_{n}, v_{n}\right)$ be a Palais-Smale sequence for $\widetilde{W}_{\Omega}$. Then there exists a solution $\left(u_{0}, v_{0}\right)$ of system $(1.11), m$ sequences of positive numbers $r_{n}^{i}(1 \leq i \leq m)$, and $m$ sequences of points $x_{n}^{i}(1 \leq i \leq m)$, when $n \rightarrow \infty$, such that up to a subsequence:

(1) $\left(u_{n}, v_{n}\right)=\left(u_{0}, v_{0}\right)+\sum_{i=1}^{m}\left(r_{n}^{i}\right)^{\frac{N-p}{p}}\left(U_{i}\left(r_{n}^{i}\left(x-x_{n}^{i}\right)\right), V_{i}\left(r_{n}^{i}\left(x-x_{n}^{i}\right)\right)\right)+\left(\sigma_{n}^{1}+\sigma_{n}^{2}\right)$,

where $r_{n}^{i} \rightarrow \infty,\left\|\left(\sigma_{n}^{1}, \sigma_{n}^{2}\right)\right\| \rightarrow 0$

(2) $J\left(u_{n}, v_{n}\right)=J\left(u_{0}, v_{0}\right)+\sum_{i=1}^{m} E^{\infty}\left(U_{i}, V_{i}\right)+o(1)$.

By Lemma 5.1, we have the existence of positive solutions of (1.11).

Theorem 5.2 Assume that $\Omega \subset \mathbb{R}^{N}$ is a bounded domain and there exists $0<R_{1}<R_{2}<$ $+\infty$ satisfying

$$
\begin{aligned}
& \left\{x \in \mathbb{R}^{N}: R_{1}<|x|<R_{2}\right\} \subset \Omega, \\
& \left\{x \in \mathbb{R}^{N}:|x|<R_{1}\right\} \not \subset \bar{\Omega} .
\end{aligned}
$$

Then problem (1.11) admits a positive solution when $\frac{R_{1}}{R_{2}}$ is sufficiently large. 
Proof Using a similar method as that in [27], let

$$
\begin{aligned}
& J_{1}(u, v)=\int_{\Omega}|\nabla u|^{p}+|\nabla v|^{p} \\
& S:=\inf _{u \in W^{1, p}\left(\mathbb{R}^{N}\right) \backslash\{0\}} \frac{\|u\|^{p}}{|u|_{p^{*}}^{p}} \quad S_{0}:=f(\tau) S,
\end{aligned}
$$

where $f(\tau):=\frac{1+\tau^{p}}{\left(1+\tau^{\beta}+\tau^{*}\right)^{\frac{p}{p^{*}}}}$.

$$
\widetilde{M}:=\left\{(u, v) \in \widetilde{W}_{\Omega}: \int_{\Omega}\left(\left(u^{+}\right)^{p^{*}}+\left(v^{+}\right)^{p^{*}}+\left(u^{+}\right)^{\alpha}\left(v^{+}\right)^{\beta}\right) d x=1\right\} .
$$

By similar methods as those in [27], if we choose $\varepsilon>0$ small enough, $J_{1}$ has no critical value in $\left(S_{0}, S_{0}+\varepsilon\right]$. However, if $J_{1}$ does not have a critical point, then the unit sphere is contractible in $\Omega$, which contradicts $\left\{x \in \mathbb{R}^{N}:|x|<R_{1}\right\} \not \subset \bar{\Omega}$. This implies that $J_{1}$ has critical points, and so the system has nontrivial positive solutions. Without loss of generality, let $R_{1}=\frac{1}{4 R}<1, R_{2}=4 R>1$. For $\forall \sigma \in S_{n}:=\left\{x \in \mathbb{R}^{N}|| x \mid=1\right\}$ (unit sphere), $0 \leq t \leq 1$, we denote

$$
u_{t}^{\sigma}(x)=\left(\frac{(1-t)^{\frac{1}{p-1}}}{(1-t)^{\frac{p}{p-1}}+(x-\sigma t)^{\frac{p}{p-1}}}\right)^{\frac{N-p}{p}} \in W^{1, p}\left(\mathbb{R}^{N}\right)
$$

In order to make $\omega_{t}^{\sigma} \in W_{0}^{1, p}(\Omega)$ for every $u_{t}^{\sigma}(x)$, and $\int_{\mathbb{R}^{N}}\left|\nabla\left(u_{t}^{\sigma}, \tau u_{t}^{\sigma}\right)-\left(\omega_{t}^{\sigma}, \tau \omega_{t}^{\sigma}\right)\right|^{p} d x$ is small enough, we choose a radially symmetric function $\phi \in C_{0}^{\infty}$ as follows:

$$
\phi_{R}(x)= \begin{cases}\phi(R x) & 0 \leq|x| \leq \frac{1}{R} \\ 1 & \frac{1}{R} \leq|x| \leq R \\ \phi\left(\frac{x}{R}\right) & |x| \geq R\end{cases}
$$

where $\phi$ satisfies: $0 \leq \phi \leq 1$, when $x \in \Omega ; \phi=1$, when $\frac{1}{2}<|x|<2 ; \phi=0$, when $x$ is not in $\frac{1}{4}<|x|<4$. So $\phi_{R}(x) \in C_{0}^{\infty}(\Omega)$, and set $\omega_{t}^{\sigma}=u_{t}^{\sigma}(x) \phi_{R}(x)$, then

$$
\begin{aligned}
& \left\|\left(u_{t}^{\sigma}, \tau u_{t}^{\sigma}\right)-\left(\omega_{t}^{\sigma}, \tau \omega_{t}^{\sigma}\right)\right\|_{\widetilde{W}\left(\mathbb{R}^{N}\right)}^{p} \\
& =\left(1+\tau^{2}\right)^{\frac{p}{2}} \int_{\mathbb{R}^{N}}\left|\nabla\left(u_{t}^{\sigma}\left(\phi_{R}-1\right)\right)\right|^{p} d x \\
& \quad \leq 2^{p}\left(1+\tau^{2}\right)^{\frac{p}{2}}\left[\int_{\mathbb{R}^{N}}\left|\nabla u_{t}^{\sigma}\right|^{p}\left(\phi_{R}-1\right)^{p}+\int_{\mathbb{R}^{N}}\left|u_{t}^{\sigma}\right|^{p}\left|\nabla \phi_{R}\right|^{p}\right] \\
& \leq C\left[\int_{B_{2 R}^{C} \cup B}\left|\nabla u_{t}^{\sigma}\right|^{p} d x+\frac{1}{R^{p}} \int_{B_{4 R} \backslash B_{2 R}}\left|\nabla u_{t}^{\sigma}\right|^{p} d x+\int_{B_{\frac{1}{2 R}}}\left|u_{t}^{\sigma}\right|^{p} d x\right. \\
& \quad:=A+B+C .
\end{aligned}
$$

Obviously, $A \rightarrow 0$, when $R \rightarrow \infty$.

$$
B \leq \frac{C}{R^{p}}\left(\int_{B_{4 R} \backslash B_{2 R}}\left|u_{t}^{\sigma}\right|^{p^{*}} d x\right) R^{1-\frac{p}{p^{*}}} \rightarrow 0 .
$$


Denote

$$
v_{t}^{\sigma}=\frac{\omega_{t}^{\sigma}}{\left\|\left(\omega_{t}^{\sigma}, \tau \omega_{t}^{\sigma}\right)\right\|_{L^{p^{*}}}},
$$

where $\left\|\left(\omega_{t}^{\sigma}, \tau \omega_{t}^{\sigma}\right)\right\|_{L^{p^{*}}}^{p^{*}}=\int_{\Omega}\left(u^{+}\right)^{p^{*}}+\left(v^{+}\right)^{p^{*}}+\left(u^{+}\right)^{\alpha}\left(v^{+}\right)^{\beta}, \lim _{R \rightarrow \infty} \int_{\mathbb{R}^{N}}\left|\nabla v_{t}^{\sigma}-\nabla \frac{u_{t}^{\sigma}}{\left\|u_{t}^{\sigma}\right\|_{L^{p^{*}}}}\right| d x \rightarrow$ $0,\left(v_{t}^{\sigma}, \tau v_{t}^{\sigma}\right)\left(v_{0}, \tau v_{0}\right) \in \tilde{M}$; hence $J_{1}\left(v_{t}^{\sigma}, \tau v_{t}^{\sigma}\right) \rightarrow S_{0}$.

Therefore, we just have to prove:

There exists $\left(u_{0}, v_{0}\right) \in \tilde{M}$ such that $J_{1}\left(u_{0}, v_{0}\right)<S_{0}+\varepsilon, J_{1}^{\prime}\left(u_{0}, v_{0}\right)=0$.

We prove the problem by reduction to absurdity. By Lemma 5.1, $J_{1}$ does not have any Palais-Smale sequence at level $\left(S_{0}, S_{0}+\varepsilon\right]$. By the deformation lemma in [21], $\exists \varepsilon>0$, and a flow $\Phi: \tilde{M} \times[0,1] \rightarrow \widetilde{M}$, such that

$$
\Phi\left(\tilde{M}_{c+\varepsilon}, 1\right) \subset \tilde{M}_{c-\varepsilon}
$$

where $\widetilde{M}_{c}=\left\{(u, v) \in \widetilde{M}: J_{1}(u, v)<c\right\}$.

By the deformation lemma, for given $\delta>0$, there exists a flow $\Phi: \widetilde{M} \times[0,1] \rightarrow \widetilde{M}$ such that

$$
\begin{aligned}
& \Phi\left(\tilde{M}_{S_{1}}, 1\right) \subset \tilde{M}_{S_{0}+\delta} \\
& \Phi((u, v), t)=(u, v), \quad \forall(u, v) \in \tilde{M}_{S_{0}+\frac{\delta}{2}} .
\end{aligned}
$$

Let

$$
J_{2}(u, v)=\int_{\Omega} x\left(\left(u^{+}\right)^{p^{*}}+\left(v^{+}\right)^{p^{*}}+\left(u^{+}\right)^{\alpha}\left(v^{+}\right)^{\beta}\right) d x
$$

By Ekeland's variational principle, there exists $x_{1} \in \bar{\Omega}$ such that, as $n \rightarrow \infty$,

$$
\begin{aligned}
& \left(\left(u_{n}^{+}\right)^{p^{*}}+\left(v_{n}^{+}\right)^{p^{*}}+\left(u_{n}^{+}\right)^{\alpha}\left(v_{n}^{+}\right)^{\beta}\right) \rightarrow \delta_{x_{1}}, \\
& |\nabla u|^{p}+|\nabla u|^{p} \rightarrow S_{0} \delta_{x_{1}} .
\end{aligned}
$$

That shows $J_{2}\left(\widetilde{M}_{S_{0}+\delta}\right) \subset U$ for any given neighborhood $U \subset \Omega$.

Assume that the projection $\mathrm{E}$ of a neighborhood $U$ of $\bar{\Omega}$ is continuous, and $E: \bar{U} \rightarrow \bar{\Omega}$. Let

$$
h: \quad S_{n} \times[0,1] \rightarrow \Omega, \quad h(\sigma, t)=E\left(J_{2}\left(\Phi\left(\left(v_{t}^{\sigma}, \tau v_{t}^{\sigma}\right), 1\right)\right)\right)
$$

because

$$
\lim _{t \rightarrow 1} \int_{\Omega}\left|\nabla v_{t}^{\sigma}\right|^{p} d x=S_{0}
$$

Hence, $v_{t}^{\sigma} \in \widetilde{M}_{S_{0+}}$, so we have

$$
\Phi\left(v_{t}^{\sigma}, 1\right)=v_{t}^{\sigma}
$$


Therefore,

$$
\lim _{t \rightarrow 1} J_{2}\left(\Phi\left(v_{t}^{\sigma}, 1\right)\right)=\lim _{t \rightarrow 1} J_{2}\left(v_{t}^{\sigma}\right)=\sigma \in S_{n} \subset \Omega
$$

It shows that $h$ is continuous and satisfies $h(\sigma, 1)=\sigma$, and for some $x_{2}$,

$$
h(\sigma, 0)=E\left(J_{2}\left(\Phi\left(v_{0}, 1\right)\right)\right)=x_{2} \in \Omega .
$$

Hence $S_{n}$ can contract to $x_{2}$ in $\bar{\Omega}$, it is a contradiction. By the strong maximum principle, we have $u_{0}>0, v_{0}>0$. So, we have completed the proof.

\section{Acknowledgements}

We would like to express our great thanks to the referees for their valuable suggestions.

\section{Funding}

This work was supported by the Fundamental Research Funds for Youth Development of the Army Engineering University of PLA. (Grant No. KYJBJQZL1926).

\section{Availability of data and materials}

Not applicable.

\section{Competing interests}

The authors declare that they have no competing interests.

\section{Authors' contributions}

All authors contributed equally and significantly in writing this article. All authors read and approved the final manuscript.

\section{Author details}

'Department of Basic Courses, The Army Engineering University of PLA, Nanjing, 211101, P.R. China. ${ }^{2}$ College of Science, Hohai University, Nanjing, 210098, P.R. China.

\section{Publisher's Note}

Springer Nature remains neutral with regard to jurisdictional claims in published maps and institutional affiliations.

Received: 2 September 2020 Accepted: 1 December 2020 Published online: 09 December 2020

\section{References}

1. Pohozaev, S.: Eigenfunctions of the equation $\Delta u+\lambda u=0$. Sov. Math. Dokl. 6, 1408-1411 (1965)

2. Brézis, H., Nirenberg, L.: Positive solutions of nonlinear elliptic equations involving critical Sobolev exponents. Commun. Pure Appl. Math. 36(4), 437-477 (2010)

3. Kawano, N., Ni, W.M., Yotsutani, S.: A generalized Pohozaev identity and its applications. J. Math. Soc. Jpn. 1990(3), 541-564 (1990)

4. Guedda, M., Veron, L.: Quasi-linear elliptic equations involving critical exponents. Nonlinear Anal. 1318, 879-902 (1989)

5. Dávila, J., Peral, I.: Nonlinear elliptic problems with a singular weight on the boundary. Calc. Var. Partial Differ. Equ. 41(3-4), 567-586 (2011)

6. Xuan, B., Wang, J.: Existence of a nontrivial weak solution to quasilinear elliptic equations with singular weights and multiple critical exponents. Nonlinear Anal. 72(9-10), 3649-3658 (2010)

7. Tai, S.J., Wang, C.H.: Existence of positive solutions for a class of degenerate or singular equations. Nonlinear Anal. 71, 1691-1698 (2009)

8. Fleckingera, J., Reichel, W.: Global solution branches for p-Laplacian boundary value problems. Nonlinear Anal. 62(1), 53-70 (2005)

9. Pucci, P., Servadei, R.: Existence, non-existence and regularity of radial ground states for p-Laplacian equations with singular weights. Ann. Inst. Henri Poincaré, Anal. Non Linéaire 25(3), 505-537 (2008)

10. Deng, Y., Wang, J.: Nonexistence of radial node solutions for elliptic problems with critical Sobolev exponents. Nonlinear Anal. 71(1), 172-178 (2009)

11. Soohyun, B., Choi, H.O., Pahk, D.H.: Existence of nodal solutions of nonlinear elliptic equations. Proc. R. Soc. Edinb., Sect. A, Math. 137(6), 1135-1155 (2007)

12. Kou, B., An, T.: Pohozaev-type inequalities and their applications for elliptic equations. Bound. Value Probl. 2017(1), 103 (2017)

13. De Thélin, F., Vélin, J.: Existence and non-existence of nontrivial solutions for some nonlinear elliptic systems. Rev. Mat. Univ. Complut. Madr. 6, 153-154 (1993)

14. Clément, Ph., Fleckinger, J., Mitidieri, E., De Thelin, F.: Existence of positive solutions for quasilinear elliptic systems. J. Differ. Equ. 166(2), 455-477 (2000) 
15. Mitidieri, E., Sweers, G., Van der Vorst, R.: Non-existence theorems for systems of quasilinear partial differential equations. Differ. Integral Equ. 8(6), 1331-1354 (1995)

16. Bozhkov, Y., Mitidieri, E.: Existence of multiple solutions for quasilinear systems via fibering method. J. Differ. Equ. 190(1), 239-267 (2003)

17. Li, G., Ye, H.: Existence of positive ground state solutions for the nonlinear Kirchhoff type equations in $\mathbb{R}^{3}$. J. Differ. Equ. 8(257), 566-600 (2014)

18. He, Y., Li, G.: Standing waves for a class of Kirchhoff type problems in $\mathbb{R}^{3}$ involving critical Sobolev exponent. Calc. Var. Partial Differ. Equ. 8(54), 3067-3106 (2015)

19. Xue, Y.F., Tang, C.L.: Existence of a bound state solution for quasilinear Schrödinger equations. Adv. Nonlinear Anal. 8(1), 323-338 (2019)

20. Pohozaev, S.I: On eigenfunctions of quasilinear elliptic problems. Math. USSR Sb. 11(2), 171-188 (1970)

21. Coron, J.: Topologie et cas limite des injections de Sobolev. C. R. Acad. Sci. Paris, Ser. I 299(1), 209-211 (1984)

22. Adachi, S., Tanaka, K.: Four positive solutions for the semilinear elliptic equation: $-\Delta u+a(x) u^{p}+f(x)$ in $\mathbb{R}^{N}$. Calc. Var. Partial Differ. Equ. 63, 63-95 (2000)

23. He, H., Yang, J.: Positive solutions for critical elliptic systems in non-contractible domains. Commun. Pure Appl. Anal. 5 , 1109-1122 (2008)

24. Lin, H.: Three positive solutions of semilinear elliptic equations in the half space with a hole. NoDEA Nonlinear Differ. Equ. Appl. 230(2), 614-633 (2006)

25. Tarantello, G.: On nonhomogeneous elliptic equations involving critical Sobolev exponent. Ann. Inst. Henri Poincaré 9(3), 281-304 (1992)

26. Han, P.: High-energy positive solutions for a critical growth Dirichlet problem in non-contractible domains. Nonlinear Anal. 60(2), 369-387 (2005)

27. Peng, S., Peng, Y., Wang, Z.: On elliptic systems with Sobolev critical growth. Calc. Var. Partial Differ. Equ. 55(6), 142 (2016)

28. An, T.: Non-existence of positive solution of some elliptic equations in positive-type domains. Appl. Math. Lett. 20 681-685 (2007)

29. Shen, Y.t., Wang, Y.J., Li, Z.X.: Modern Variational Methods for Quasilinear Elliptic Equations, 1st edn. Higher Education Press, Peking (2017) (in Chinese)

\section{Submit your manuscript to a SpringerOpen ${ }^{\circ}$ journal and benefit from:}

- Convenient online submission

- Rigorous peer review

- Open access: articles freely available online

- High visibility within the field

- Retaining the copyright to your article

Submit your next manuscript at $\boldsymbol{~ s p r i n g e r o p e n . c o m ~}$ 